\title{
BMJ Open Competing-risks nomogram for predicting cancer-specific death in upper tract urothelial carcinoma: a population- based analysis
}

\author{
Chengzhuo Li, ${ }^{1,2}$ Didi Han, ${ }^{1,2}$ Qiao Huang, ${ }^{3}$ Fengshuo Xu, ${ }^{1,2}$ Shuai Zheng, ${ }^{1,4}$ \\ Xiang Li, ${ }^{5}$ Fanfan Zhao, ${ }^{1,2}$ Xiaojie Feng, ${ }^{1,2}$ Jun Lyu (D) ${ }^{1,2}$
}

To cite: Li C, Han D, Huang Q, et al. Competing-risks nomogram for predicting cancer-specific death in upper tract urothelial carcinoma: a populationbased analysis. BMJ Open 2021;11:e048243. doi:10.1136/ bmjopen-2020-048243

- Prepublication history for this paper is available online. To view these files, please visit the journal online (http://dx.doi org/10.1136/bmjopen-2020048243).

Received 22 December 2020 Accepted 25 June 2021

Check for updates

(C) Author(s) (or their employer(s)) 2021. Re-use permitted under CC BY-NC. No commercial re-use. See rights and permissions. Published by BMJ.

${ }^{1}$ Department of Clinical Research, Jinan University First Affiliated Hospital, Guangzhou, Guangdong, China

${ }^{2}$ School of Public Health, Xi'an Jiaotong University, Xi'an,

Shaanxi, China

${ }^{3}$ Department of Chemotherapy and Radiation Therapy, Wuhan University Zhongnan Hospital,

Wuhan, Hubei, China

${ }^{4}$ Shaanxi University of Chinese

Medicine, Xianyang, China

${ }^{5}$ Xian Jiaotong University, Xi'an, China

Correspondence to

Dr Jun Lyu;

lyujun2020@jnu.edu.cn

\section{ABSTRACT}

Objective This study aimed to use a competing-risks model to establish a nomogram to accurately analyse the prognostic factors for upper tract urothelial carcinoma (UTUC) cancer-specific death (CSD).

Design Retrospective observational cohort study. Setting The programme has yielded a database of all patients with cancer in 18 defined geographical regions of the USA.

Participants We selected patients with UTUC from the latest edition of the Surveillance, Epidemiology, and End Results database from 1975 to 2016. After excluding patients with unknown histological grade, tumour size and lymph node status, 2576 patients were finally selected.

Primary and secondary outcome measures We used the Fine-Gray proportional subdistribution hazards model for multivariate analysis and compared the results with cause-specific hazards model. We finally constructed a nomogram for 3-year, 5-year and 8-year CSD rates and tested these rates in a validation cohort.

Results The proportional subdistribution hazards model showed that sex, tumour size, distant metastasis, surgery status, number of lymph nodes positive (LNP) and lymph nodes ratio (LNR) were independent prognostic factors for CSD. All significant factors associated with CSD were included in the nomogram. The 3-year, 5-year and 8-year concordance indexes were $0.719,0.702$ and 0.692 in the training cohort and $0.701,0.675$ and 0.668 in the validation cohort, respectively.

Conclusions The competing-risks model showed that sex, tumour size, distant metastasis, surgery status, LNP and LNR were associated with CSD. The nomogram predicts the probability of CSD in patients with UTUC at 3, 5 and 8 years, which may help clinicians in predicting survival probabilities in individual patients.

\section{INTRODUCTION}

Urothelial carcinomas are the fourth most common type of tumour, ${ }^{1}$ which is located in the upper or lower urinary tract. Upper tract urothelial carcinoma (UTUC), including the renal pelvis and ureter carcinoma, currently accounts for $5 \%$ of urothelial malignancies. ${ }^{2}$ The annual incidence of UTUC is typically
Strengths and limitations of this study

- The study established the first competing-risks nomogram for predicting the 3-year, 5-year and 8-year specific mortality probability for upper tract urothelial carcinoma (UTUC) based on a large retrospective sample, which can improve the ability of clinicians in predicting survival probabilities in individual patients.

- The established model is not comprehensive enough because the Surveillance, Epidemiology, and End Results database does not include all prognostic factors for UTUC.

- The data available on treatment status are not sufficiently detailed to distinguish the impact of various treatment plans.

- The model requires prospective studies to confirm its reliability.

estimated at 1 or 2 per 100000 inhabitants in Western countries. ${ }^{3}$ However, the increasing morbidity and mortality associated with $\mathrm{UTUC}^{45}$ make research more important.

A previous study showed that UTUC has unique prognostic factors which are different from bladder cancer and other urinary tract cancers. ${ }^{6}$ Most studies analysing the prognostic factors for UTUC have adopted the Kaplan-Meier (K-M) method or Cox regression methods. ${ }^{7-9}$ These methods only consider a single endpoint while determining survival parameters. However, in clinical research, in addition to events of interest, there are often competing events. Competing events for cancer deaths refer to death from other causes unrelated to primary cancer, such as other diseases, car collisions and suicide. In traditional survival analysis methods, these events were considered censored, making the cumulative incidence of cancer deaths overestimated. Applying standard survival analysis to competing-risks 
data leads to false and biased results. ${ }^{10}$ Although the use of all-cause death as the study endpoint does not cause a competing-risk bias, such an analysis cannot reflect the influence of factors on the specific endpoint of cancer death. Therefore, the cumulative incidence function (CIF) of UTUC cancer-specific death (CSD) needs to be calculated and the prognostic factors for UTUC analysed using the Fine-Gray proportional subdistribution hazards model. ${ }^{11}$

A nomogram is based on a prognostic model and graphically represents the predictive abilities of different prognostic factors as the length of line segments. This format makes it easy for clinicians to make rapid and comprehensive decisions and predict the probability of CSD, and has great clinical significance. Some studies have constructed competing-risks nomograms for cancers such as sarcoma and prostate cancer ${ }^{12}{ }^{13}$; however, there is a lack of studies related to UTUC.

The purpose of our research was to identify the prognostic factors for UTUC based on the competing-risks model and use them to construct a nomogram to predict the survival rates of patients at 3,5 and 8 years.

\section{METHODS}

\section{Database and patients}

The Surveillance, Epidemiology, and End Results (SEER) program has yielded a database of all patients with cancer in 18 defined geographical regions of the USA collected by the National Cancer Institute. It is the largest cancer registry in the USA and includes information on approximately $28 \%$ of the US population. The SEER research data are publicly available; therefore, no informed consent or institutional review board approval is required when analysing the data. We additionally requested chemotherapy data for inclusion in our research and obtained a licence for using the SEER software. $^{1415}$

We selected patients with UTUC from the latest edition of the SEER database from 1975 to 2016. The primary sites were extracted using the SEER codes 'C65.9-Renal pelvis' and 'C66.9-Ureter'. Patients between 2004 and 2015 were included in the study. We included all of the histological subtypes of UTUC, according to the Third Revision of the International Classification of Diseases for Oncology. The following demographic indicators were selected: age at diagnosis, sex, race and marital status. Primary site, histological grade, tumour size, laterality, distant metastasis, surgery status, radiotherapy status, chemotherapy status, number of lymph nodes examined (LNE), lymph nodes positive (LNP) and lymph nodes ratio (LNR; calculated as the number of LNP divided by LNE) were also included as pathological characteristics. Tumour size was divided into three groups: $<2,2-4$ and $\geq 4 \mathrm{~cm} .{ }^{16}$ The study outcomes included CSD and death due to other causes (DOC). Survival time was reported in the available data in months.

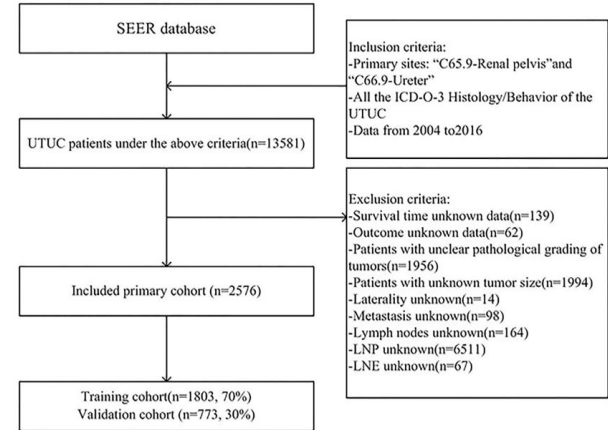

Figure 1 Data selection flow chart. ICD-O-3, Third Revision of the International Classification of Diseases for Oncology; SEER, Surveillance, Epidemiology, and End Results; UTUC, upper tract urothelial carcinoma; LNP, lymph nodes positive; LNE, lymph nodes examined.

\section{Exclusion criteria}

Our preliminary selection of the above methods initially identified 13581 patients. Then, to ensure the study's accuracy, the following were excluded: unknown histological grade, unknown tumour size and unknown lymph node status. The specific data selection process is shown in figure 1 . We finally chose 2576 patients for inclusion in the follow-up investigations.

\section{Statistical methods}

We randomly divided the 2576 eligible patients into two groups using R software (V.3.5.3, The R Foundation for Statistical Computing, Vienna, Austria; http://www.rproject.org): $70 \%(\mathrm{n}=1803)$ in the training cohort and $30 \%(\mathrm{n}=773)$ in the validation cohort. We first described the basic composition of each factor in the two patient cohorts using the R software. Age, LNE, LNP and LNR were expressed as median and IQR values, while categorical variables were presented as percentages. We evaluated differences in patient characteristics between the two groups using the Student's t-test and $\chi^{2}$ test.

We used the CIF to describe the probability of each event and also plotted the corresponding CIF curves. Moreover, Gray's test was used for univariate analysis to estimate the difference in CIF between the groups. Significant variables $(p<0.05)$ were included in the multivariate regression model. The Fine-Gray proportional subdistribution hazards model was used for multivariate analysis and compared with the results of the cause-specific hazards model. Applying the standard Cox regression method ignores the presence of competing risks and hence overestimates the actual incidence of beneficial events, leading to inappropriate risk stratification. ${ }^{17}$ Several studies have confirmed that different approaches can be used in competing-risks settings for multivariate survival analysis. However, proportional subdistribution hazards model is the best method to predict the survival probability. ${ }^{18-20}$

Finally, the results of Fine-Gray proportional subdistribution hazards model were used to construct a nomogram of the 3-year, 5-year and 8-year CSD rates. We used 
the concordance index (C-index) and calibration plots to evaluate the differentiation ability and consistency of the established model in the training and validation cohorts.

All statistical tests were conducted using $\mathrm{R}$ software (V.3.5.3). Probability values of $\mathrm{p}<0.05$ were considered statistically significant and all tests were two-sided. The SEER database can be accessed free of charge. This study was exempted from obtaining informed consent.

\section{Patient and public involvement}

This study was conducted without patient involvement. Patients were not invited to comment on the study design and were not consulted to develop patient-relevant outcomes or interpret the results. Moreover, patients were not allowed to contribute to the writing or editing of this document for readability or accuracy.

\section{RESULTS}

\section{Patient characteristics}

The composition of each variable for the 2576 patients in the training and validation cohorts is presented in table 1 . The median age was 71 years in the training and validation cohorts, respectively. The majority of patients were male $(60.6 \%$ and $57.4 \%)$, white $(86.2 \%$ and $82.5 \%)$ and married $(86.8 \%$ and $87.6 \%)$. The main sites of UTUC were in the renal pelvis $(63.9 \%$ and $62.7 \%$, respectively, in the training and validation cohorts), with the rest in the ureter. The majority of patients were in the undifferentiated stage $(58.1 \%$ and $55.6 \%$ ), and most of the tumours in both cohorts were larger than $4 \mathrm{~cm}$. Most patients in both cohorts had received surgery, whereas a few patients had received radiotherapy or chemotherapy. Only about $9 \%$ of the patients had distant metastasis. The baseline characteristics of the training cohorts and validation cohorts were similar.

\section{Univariate analysis}

We calculated the 3-year, 5-year and 8-year cumulative incidence rates of CSD and DOC. Year, laterality and marital status were not related to either outcome $(p>0.05)$, while age, sex, histological grade, chemotherapy status and LNR were related to both outcomes $(\mathrm{p}<0.05)$. Race, primary site, tumour size, surgery status, radiotherapy status, distant metastasis, LNE and LNP were significantly related to CSD. The corresponding CIF curves are shown in figure 2. The cumulative incidences of CSD and DOC are compared in table 2.

\section{Multivariate analysis}

Table 3 shows the comparison of the proportional subdistribution hazards model with the cancer-specific hazards model. The cancer-specific hazards model showed that sex, tumour size, distant metastasis, LNP and LNR were prognostic factors for UTUC $(\mathrm{p}<0.001)$. We constructed the Fine-Gray proportional subdistribution hazards model, which indicates that sex (HR=1.480 for female, $95 \%$ CI 1.241 to 1.764$)$, tumour size ( $\mathrm{HR}=1.556$ for
$2-4 \mathrm{~cm}, 95 \%$ CI 1.092 to 2.216 ; HR=2.205 for $\geq 4 \mathrm{~cm}, 95 \%$ CI 1.575 to 3.087 ), surgery status ( $\mathrm{HR}=2.205$ for no/ unknown surgery, 95\% CI 1.292 to 3.761), distant metastasis (HR=2.414 for distant, 95\% CI 1.842 to 3.163$)$, LNP $(\mathrm{HR}=1.064,95 \%$ CI 1.022 to 1.107$)$ and $\mathrm{LNR}(\mathrm{HR}=1.873$, $95 \%$ CI 1.435 to 2.445 ) were prognostic factors affecting UTUC, as shown in table 3 .

\section{Construction and verification of the nomogram}

Figure 3 shows the nomogram constructed according to the results of the Fine-Gray proportional subdistribution hazards model for predicting CSD probabilities at 3, 5 and 8 years. LNP had the most significant impact on the probability of CSD, followed by distant metastasis, tumour size, LNR, surgery and sex (figure 3 ).

We used both the training and validation cohorts to verify the nomogram after establishing it. The 3-year, 5-year and 8-year C-indexes were 0.719, 0.702 and 0.692 for the training cohort, respectively, and 0.701, 0.675 and 0.668 for the validation cohort. All of these values exceeded 0.6, indicating that the model had good discrimination ability. We then tested the prediction accuracy of the model. As shown in figure 4, the 3-year, 5-year and 8-year calibration plots for both cohorts were very close to the standard straight line, demonstrating that the model was well calibrated.

\section{DISCUSSION}

The increasing incidence of UTUC ${ }^{21}$ makes it necessary to further explore the prognostic factors for UTUC. The present study used a competing-risks model to accurately explore the prognostic factors for UTUC. The study used these factors to construct a nomogram that provides clinicians with direct guidance while making relevant decisions.

The application of the study criteria resulted in the inclusion of 2576 patients from the SEER database, and 1542 of these patients died during follow-up. However, only 750 of the deaths were related to UTUC. These results indicate that the number of DOC patients was almost the same as that for CSD. In this situation, if the traditional K-M or Cox survival analysis had been adopted, the DOC patients will be regarded as censored. This will lead to an overestimation of the cumulative incidence of CSD, which cannot truly reflect the prognosis. ${ }^{22}{ }^{23}$ We overcame this shortcoming by using a competing-risks model which can adequately address the situation where the available data are related to multiple potential outcomes. ${ }^{24}$ This method was first proposed by Fine and Gray and applied in previous studies. ${ }^{172526}$ In the case of competing risks, there are usually two models. One is the cause-specific hazards model (CS), and the other is the proportional subdistribution hazards model (SD), also known as the Fine-Gray model. In the present study, two models were analysed and compared. CS is suitable for answering aetiological questions, and SD is suitable for establishing clinical prediction models and risk scores. Therefore, we 
Open access

Table 1 Basic characteristics of patients in this study

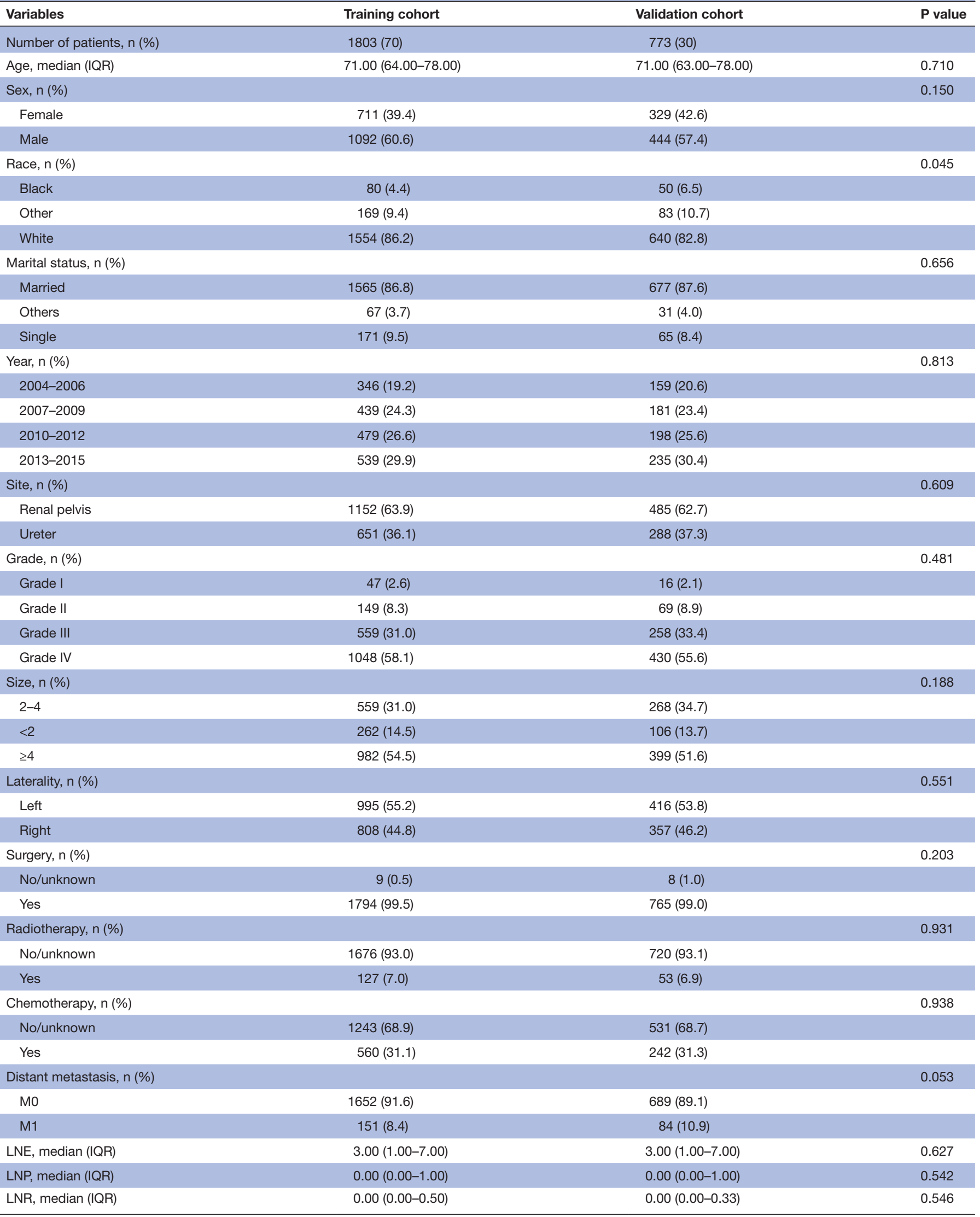

LNE, lymph nodes examined; LNP, lymph nodes positive; LNR, lymph nodes ratio. 

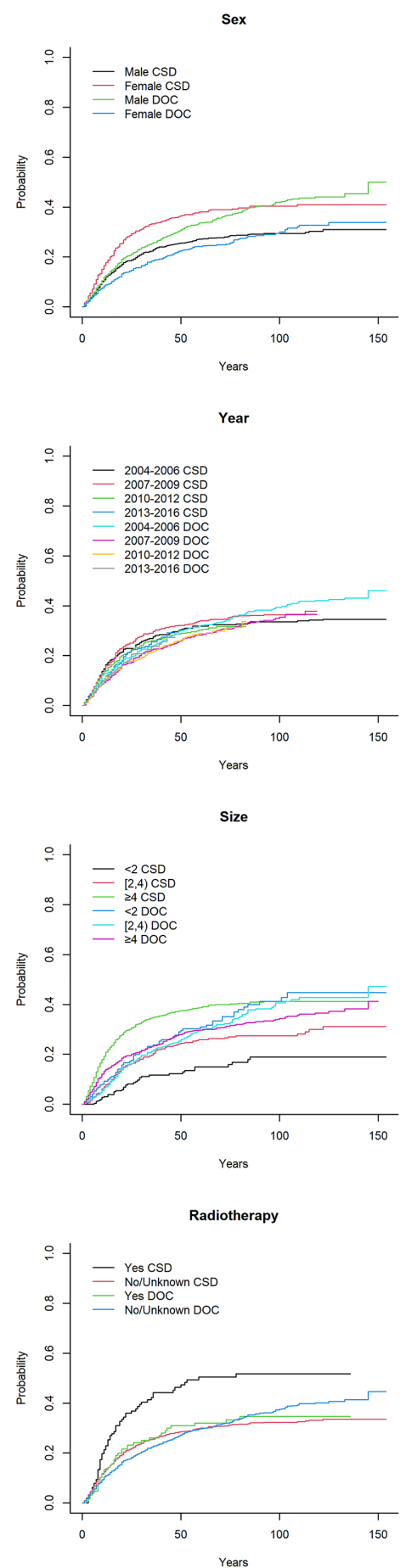

Race
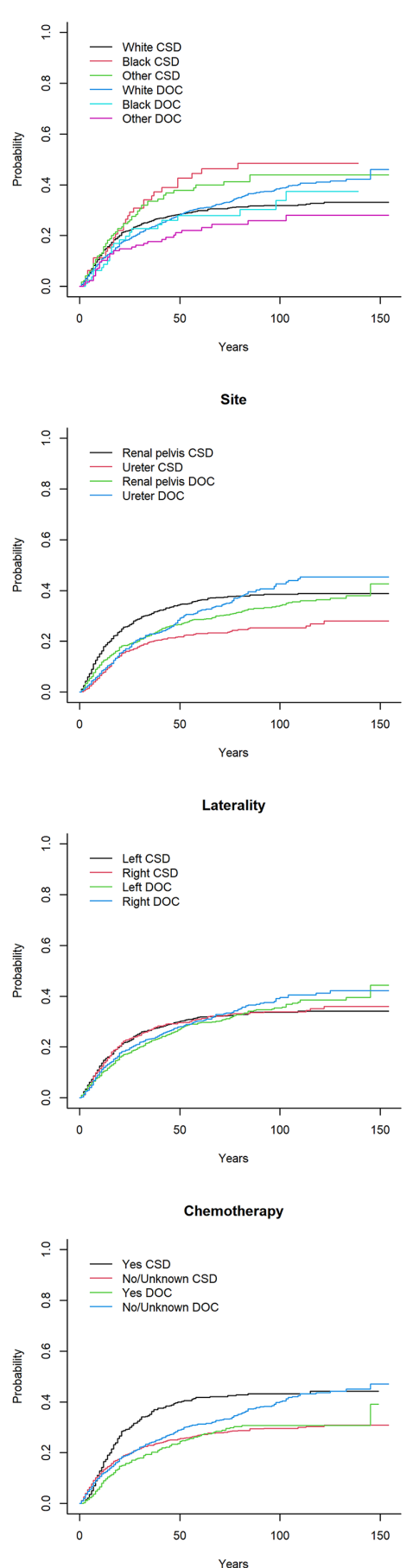

Marital
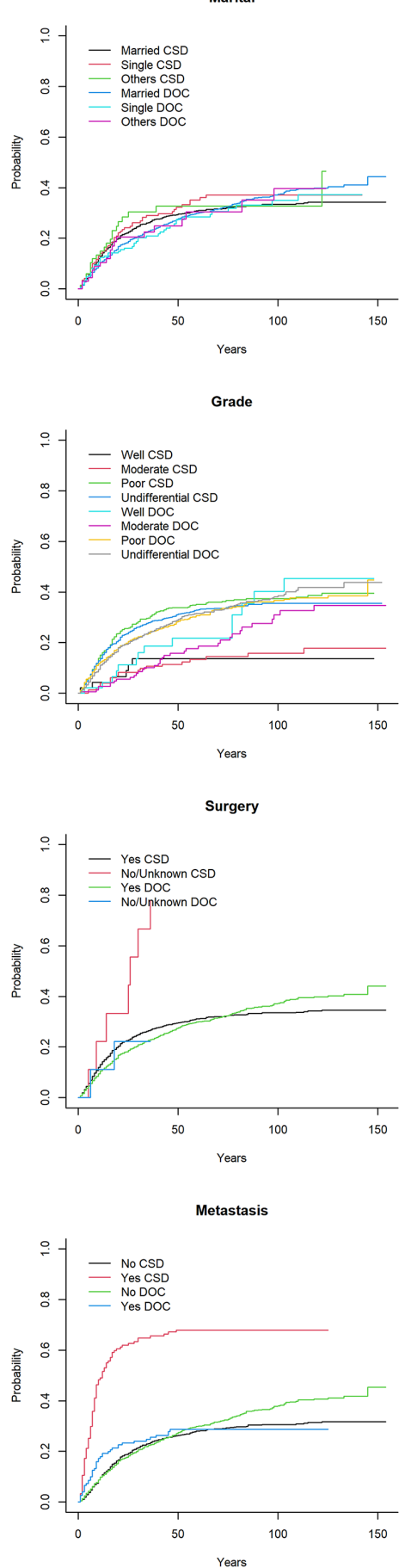

Figure 2 CIF curves of CSD and DOC among patients with upper tract urothelial carcinoma. CIF, cumulative incidence function; CSD, cancer-specific death; DOC, death due to other causes.

used the CIF and the proportional SD model to explore the impact of various factors on the prognosis of CSD.

The results of the univariate analysis showed that age, sex, race, primary site, histological grade, tumour size, surgery status, radiotherapy status, chemotherapy status, distant metastasis, LNE, LNP and LNR were influencing factors for CSD, while age, sex, histological grade, chemotherapy status and LNR were influencing factors for DOC. The results of the CS model showed that age, sex, histological grade, tumour size, distant metastasis, LNP and LNR were prognostic factors for CSD. The proportional SD model showed that sex, tumour size, surgery, distant metastasis, LNP and LNR are independent prognostic factors for CSD.

Age is generally considered to be a prognostic factor for most cancers and also for UTUC. ${ }^{27} 28$ Our CS model showed that age was a predictor of CSD; however, it was not statistically significant in the SD model. This may be because the effect of age on DOC is higher than the CSD; namely, elderly patients are more likely to die of other 


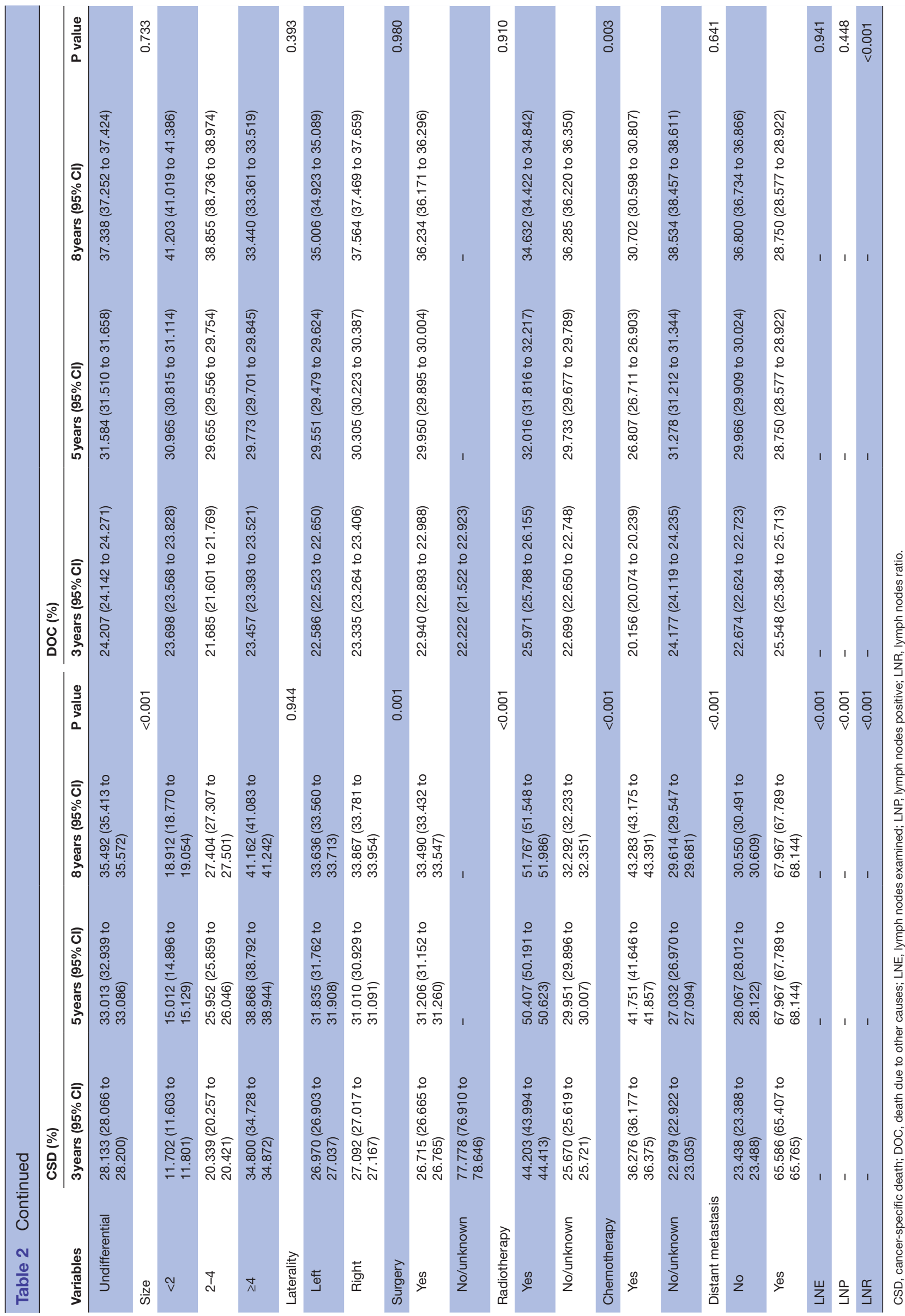

\begin{tabular}{l}
0 \\
2 \\
2 \\
0 \\
0 \\
0 \\
\hline \\
\hline
\end{tabular}

寻

등

बึ

क

$\vec{\circ}$

$\vec{\sigma}$

응.

กิ

옹

离

욱

$\overrightarrow{0}$

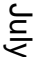

N

인 
Table 3 Multivariate analysis by proportional subdistribution hazards model and cause-specific hazards model for CSD among patients with upper tract urothelial carcinoma

\begin{tabular}{|c|c|c|c|c|c|c|c|c|}
\hline \multirow[b]{2}{*}{ Variables } & \multicolumn{4}{|c|}{ Proportional subdistribution hazards model } & \multicolumn{4}{|c|}{ Cause-specific hazards model } \\
\hline & Coefficient & sdHR & $95 \% \mathrm{Cl}$ & $P$ value & Coefficient & csHR & $95 \% \mathrm{Cl}$ & $P$ value \\
\hline Age & -0.004 & 0.996 & 0.987 to 1.005 & 0.340 & 0.009 & 1.009 & 1.000 to 1.018 & 0.039 \\
\hline Male & Reference & & & & Reference & & & \\
\hline Female & 0.392 & 1.480 & 1.241 to 1.764 & $<0.001$ & 0.301 & 1.351 & 1.134 to 1.611 & $<0.001$ \\
\hline White & Reference & & & & Reference & & & \\
\hline Black & 0.242 & 1.274 & 0.873 to 1.858 & 0.210 & 0.348 & 1.416 & 0.990 to 2.027 & 0.057 \\
\hline Other & 0.201 & 1.223 & 0.930 to 1.607 & 0.150 & 0.164 & 1.178 & 0.899 to 1.544 & 0.235 \\
\hline \multicolumn{9}{|l|}{ Site } \\
\hline Renal pelvis & Reference & & & & Reference & & & \\
\hline Moderate & -0.034 & 0.966 & 0.398 to 2.343 & 0.940 & 0.009 & 1.009 & 0.407 to 2.502 & 0.985 \\
\hline Poor & 0.763 & 2.145 & 0.971 to 4.739 & 0.059 & 0.908 & 2.479 & 1.097 to 5.601 & 0.029 \\
\hline Undifferential & 0.658 & 1.931 & 0.878 to 4.245 & 0.100 & 0.772 & 2.165 & 0.961 to 4.875 & 0.062 \\
\hline \multicolumn{9}{|l|}{ Size } \\
\hline$<2$ & Reference & & & & Reference & & & \\
\hline $2-4$ & 0.442 & 1.556 & 1.092 to 2.216 & 0.014 & 0.414 & 1.513 & 1.043 to 2.196 & 0.029 \\
\hline$\geq 4$ & 0.791 & 2.205 & 1.575 to 3.087 & $<0.001$ & 0.881 & 2.414 & 1.691 to 3.447 & $<0.001$ \\
\hline \multicolumn{9}{|l|}{ Surgery } \\
\hline Yes & Reference & & & & Reference & & & \\
\hline No/unknown & 0.025 & 1.025 & 0.829 to 1.269 & 0.820 & 0.171 & 1.187 & 0.972 to 1.450 & 0.093 \\
\hline \multicolumn{9}{|c|}{ Distant metastasis } \\
\hline No & Reference & & & & Reference & & & \\
\hline Yes & 0.881 & 2.414 & 1.842 to 3.163 & $<0.001$ & 1.252 & 3.497 & 2.741 to 4.460 & $<0.001$ \\
\hline LNE & -0.012 & 0.988 & 0.971 to 1.006 & 0.200 & -0.013 & 0.987 & 0.972 to 1.002 & 0.091 \\
\hline LNP & 0.062 & 1.064 & 1.022 to 1.107 & 0.002 & 0.069 & 1.072 & 1.032 to 1.113 & $<0.001$ \\
\hline LNR & 0.627 & 1.873 & 1.435 to 2.445 & $<0.001$ & 0.934 & 2.544 & 1.965 to 3.294 & $<0.001$ \\
\hline
\end{tabular}

CSD, cancer-specific death; csHR, cause-specific HR; LNE, lymph nodes examined; LNP, lymph nodes positive; LNR, lymph nodes ratio; sdHR, subdistribution HR.

causes, which competitively leads to the fact that CSD incidence does not increase significantly with age. Sex and race have always been controversial prognostic factors. A previous study showed that race was a preoperative prognostic factor for patients with UTUC. ${ }^{29}$ Moreover, another study found no statistically significant differences in survival between men and women. ${ }^{30}$ However, the competing-risks model in our study showed that sex was a risk factor for UTUC, while race was not. This may be because previous studies ignored the effect of competing risks. However, since most of the patients included in the SEER database are white, studies on different races need to be conducted.

Tumour size is also considered to be related to cancer prognosis. One study found 5-year recurrence-free survival rates of $46.9 \%$ and $25.8 \%$ in patients with UTUC with tumour sizes $<3 \mathrm{~cm}$ and $\geq 3 \mathrm{~cm}$, respectively. ${ }^{31}$ The univariate and multivariate analyses performed in the 
Points

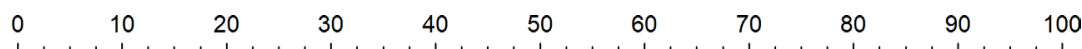

Sex

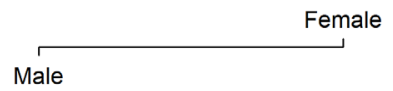

Size

Surgery

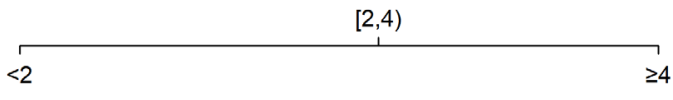

Surgery

Metastasis

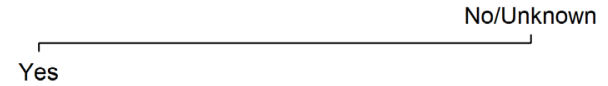

Yes

LNP

No

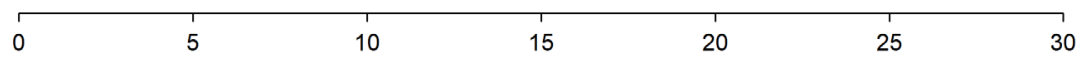

LNR

Total Points

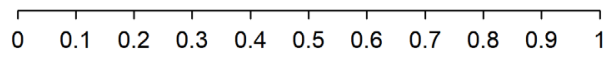

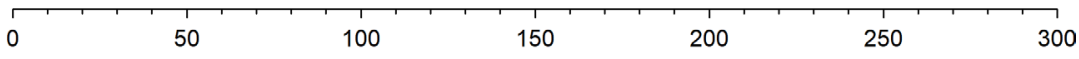

3-year CSD Prob.

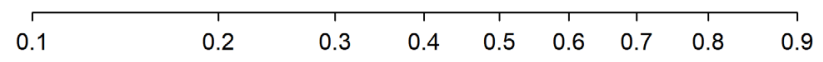

5-year CSD Prob.

$\begin{array}{lllllllll}0.1 & 0.2 & 0.3 & 0.4 & 0.5 & 0.6 & 0.7 & 0.8 & 0.9\end{array}$

8-year CSD Prob.

\begin{tabular}{lllllllll}
\hline 0.1 & 0.2 & 0.3 & 0.4 & 0.5 & 0.6 & 0.7 & 0.8 & 0.9
\end{tabular}

Figure 3 Nomogram based on the competing-risks analysis to predict CSD probabilities at 3, 5 and 8 years for patients with upper tract urothelial carcinoma. CSD, cancer-specific death; LNP, lymph nodes positive; LNR, lymph nodes ratio.

present study also indicated that tumour size was an influencing factor for CSD. The prognosis was worse for tumours larger than $2 \mathrm{~cm}$. In addition, our research also found that distant metastasis was an important risk factor for CSD. In terms of treatment methods, our study suggested that surgery status was a significant prognostic
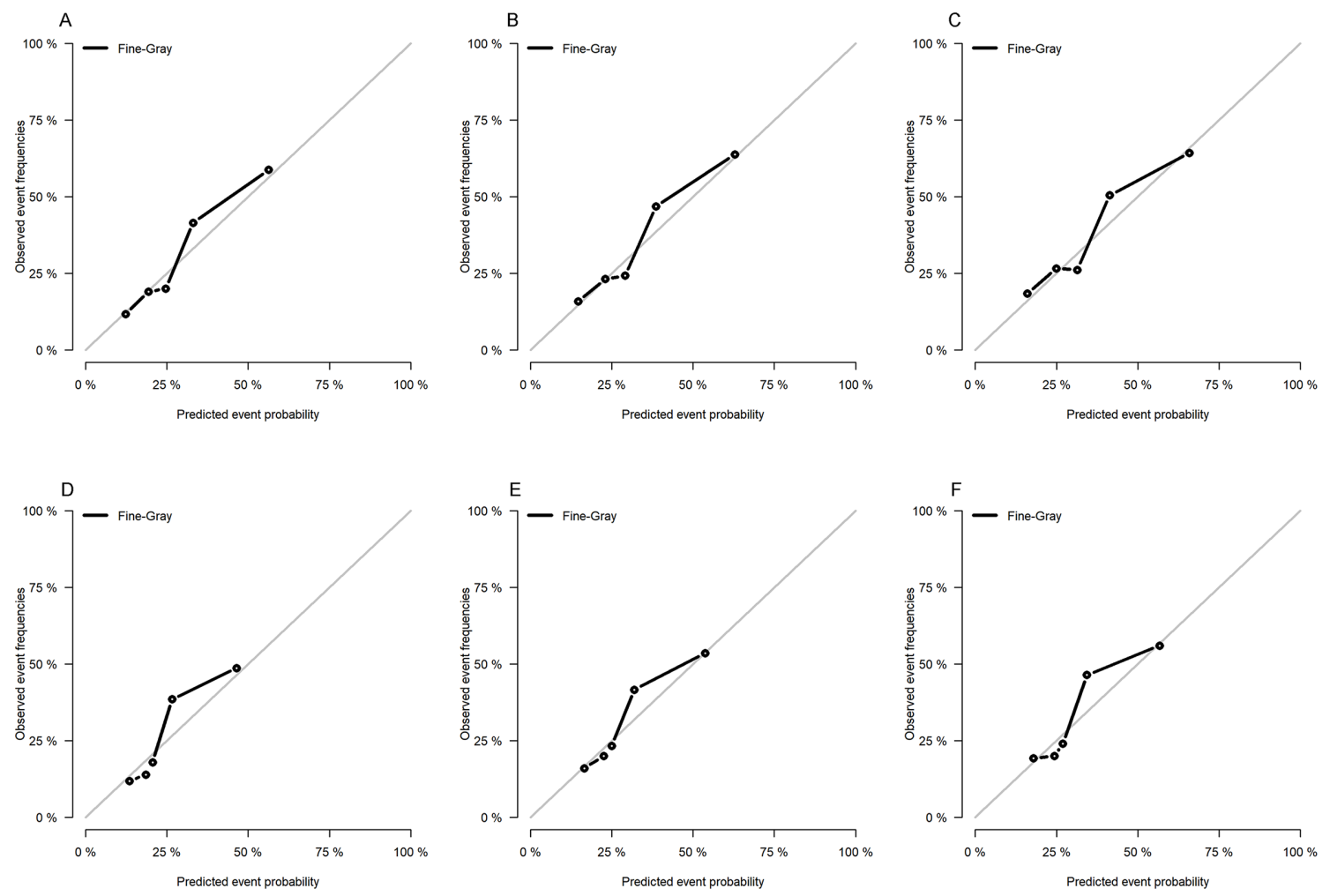

Figure 4 Calibration curves at 3, 5 and 8 years for the training $(A, B, C)$ and validation $(D, E, F)$ cohorts. 
factor, which was consistent with the findings of Freifeld et $a l .{ }^{32}$ Surgery has long been considered the gold standard of UTUC treatment. However, radiotherapy status and chemotherapy status were not influencing factors for CSD in both competing-risks models. This result conflicted with some previous findings, ${ }^{33-35}$ suggesting that traditional Cox regression analysis overestimated the effects of radiotherapy and chemotherapy. Obviously, the relative lack of information on the radiotherapy status and chemotherapy status in the SEER database may also lead to inaccurate results, and thus further exploration of these indicators is needed.

Some indicators related to lymph nodes (eg, distant lymph node metastasis, LNP and LNE) are important clinical information for cancer prognosis, but whether they are independent prognostic factors for UTUC has not been determined. One study found that lymph node metastases were significantly associated with reduced cancer-specific survival in univariate analysis. ${ }^{36}$ It is worth noting that very few studies have investigated LNP, LNE and LNR. Our study is the first to use the SEER database to analyse the prognostic impact of these indicators on UTUC, and the results may be more accurate than those involving small samples. LNR is an emerging indicator that has been regarded as a prognostic factor in rectal cancer and breast cancer. ${ }^{37} 38$ Our results also suggested that LNR was an important prognostic factor for UTUC. We found that LNE was an influencing factor for UTUC in the univariate analysis but not in the multivariate analysis. Moreover, both LNR and LNP entered the proportional SD model, suggesting that after adjusting for the effects of LNR and LNP, LNE was no longer an independent prognostic indicator. LNP was a prognostic factor in all of the analyses, indicating that it significantly influences the prognosis of UTUC.

We used the results from the above-mentioned proportional SD model to construct a nomogram that graphically represents the degree of influence of various prognostic factors. This nomogram can be used to predict the 3-year, 5-year and 8-year probabilities of CSD in patients with UTUC. The predictive function of the nomogram has been used for different types of cancer and has even been proposed as a new standard. The nomogram is easy to use. In order to calculate the CSD probability of a patient with UTUC, find the patient's sex (male or female) on the sex row, draw a vertical line on the dot row and obtain the sex score value. Repeat these steps for tumour size, M stage, surgery, LNP and LNR. Add the score values of each variable, find the total point on the total point axis, and draw a straight downward line to get the 3-year, 5-year and 8-year CSD probability of the patient with UTUC. For example, a woman (30 points) with a tumour size of $1.5 \mathrm{~cm}$ ( 0 points) at M1 stage (68 points) and had performed surgery (0 points), with an LNP equal to 5 (15 points) and LNR equal to 0.8 (45 points), and with a total score of 158 points, corresponds to 3-year, 5-year and 8-year CSD probability of 58\%, $64 \%$ and $69 \%$, respectively.
The C-indexes for the nomogram all exceeded 0.6, demonstrating that the model provided a good fit to the available data. The prediction calibration curves in figure 4 were very close to the standard curve, indicating that the nomogram had good predictive ability. The results for the validation cohort also showed that the model was stable. Therefore, this model can help clinicians to quickly and easily determine the prognosis of individual patients and provide guidance in their clinical decision-making. However, the stability of the model needs further verification.

Our study used the large sample size and high-quality data from SEER database and competing-risks model, which guaranteed the accuracy of our study. However, inevitably, our research had some limitations. First, the established model is not comprehensive enough because the SEER database does not include all prognostic factors for UTUC. Second, the data available on treatment status are not sufficiently detailed to distinguish the impact of various treatment plans. Third, as a retrospective study, our results may be affected by confounding bias to some extent, so the conclusion needs to be further verified in future prospective studies. Fourth, Some of the causes of death in SEER may have been misclassified according to the death certificate report, which may also bring information bias to our study.

\section{CONCLUSIONS}

In summary, this study used a competing-risks model to determine the prognostic factors for UTUC. The proportional subdistribution hazards model showed that sex, tumour size, surgery, distant metastasis, LNP and LNR were associated with CSD, while LNE was not. The constructed nomogram can predict the 3-year, 5-year and 8-year CSD probabilities of patients based on these relevant factors, which can support clinicians in making better decisions on the survival rates of individual patients.

Acknowledgements The authors would like to thank two referees and the associate editor for their constructive advice.

Contributors $\mathrm{JL}, \mathrm{CL}$ and SZ designed the study. QH, DH and FX collected and analysed the data. $C L$ and XL drafted the initial manuscript. FZ and XF reviewed and edited the article. All authors read and approved the final manuscript.

Funding The study was supported by the National Social Science Foundation of China (grant no. 16BGL183).

Competing interests None declared.

Patient and public involvement Patients and/or the public were not involved in the design, or conduct, or reporting, or dissemination plans of this research.

Patient consent for publication Not required.

Ethics approval All procedures performed in the present study were in accordance with the principles outlined in the 1964 Helsinki Declaration and its later amendments. Institutional review board approval and informed consent were not required in the current study because SEER research data are publicly available and all patient data are de-identified.

Provenance and peer review Not commissioned; externally peer reviewed. Data availability statement No data are available.

Open access This is an open access article distributed in accordance with the Creative Commons Attribution Non Commercial (CC BY-NC 4.0) license, which 
permits others to distribute, remix, adapt, build upon this work non-commercially, and license their derivative works on different terms, provided the original work is properly cited, appropriate credit is given, any changes made indicated, and the use is non-commercial. See: http://creativecommons.org/licenses/by-nc/4.0/.

ORCID iD

Jun Lyu http://orcid.org/0000-0002-2237-8771

\section{REFERENCES}

1 Rouprêt M, Babjuk M, Compérat E, et al. European association of urology guidelines on upper urinary tract urothelial carcinoma: 2017 update. Eur Urol 2018;73:111-22.

2 Munoz JJ, Ellison LM. Upper tract urothelial neoplasms: incidence and survival during the last 2 decades. J Urol 2000;164:1523-5.

3 Rouprêt M, Babjuk M, Compérat E, et al. European association of urology guidelines on upper urinary tract urothelial cell carcinoma: 2015 update. Eur Urol 2015;68:868-79.

4 Siegel RL, Miller KD, Jemal A. Cancer statistics, 2019. CA Cancer J Clin 2019;69:7-34.

5 Raman JD, Messer J, Sielatycki JA, et al. Incidence and survival of patients with carcinoma of the ureter and renal pelvis in the USA, 1973-2005. BJU Int 2011;107:1059-64.

6 Green DA, Rink M, Xylinas E, et al. Urothelial carcinoma of the bladder and the upper tract: disparate twins. J Urol 2013;189:1214-21.

7 Lughezzani G, Jeldres $\mathrm{C}$, Isbarn $\mathrm{H}$, et al. A critical appraisal of the value of lymph node dissection at nephroureterectomy for upper tract urothelial carcinoma. Urology 2010;75:118-24.

8 Novara G, Matsumoto K, Kassouf W, et al. Prognostic role of lymphovascular invasion in patients with urothelial carcinoma of the upper urinary tract: an international validation study. Eur Urol 2010;57:1064-71.

9 Remzi M, Haitel A, Margulis V, et al. Tumour architecture is an independent predictor of outcomes after nephroureterectomy: a multi-institutional analysis of 1363 patients. BJU Int 2009;103:307-11.

$10 \mathrm{Kim}$ HT. Cumulative incidence in competing risks data and competing risks regression analysis. Clin Cancer Res 2007;13:559-65.

11 Austin PC, Fine JP. Practical recommendations for reporting Fine-Gray model analyses for competing risk data. Stat Med 2017;36:4391-400.

12 Kattan MW, Heller G, Brennan MF. A competing-risks nomogram for sarcoma-specific death following local recurrence. Stat Med 2003;22:3515-25.

13 Abdollah F, Sun M, Schmitges J, et al. Cancer-Specific and othercause mortality after radical prostatectomy versus observation in patients with prostate cancer: competing-risks analysis of a large North American population-based cohort. Eur Urol 2011;60:920-30.

14 About the SEER Program. National Cancer Institute; n.d Fall. Surveillance, epidemiology, and end results program: turning cancer data into discovery, 2014. Available: http:// seer. cancer.gov/about/ overview.html

15 Yang J, Liu QQ, Geng $\mathrm{H}$. SEER database application and data extraction methods and processes. Chinese Journal of EvidenceBased Cardiovascular Medicine 2018;10:781-4.

16 Pieras E, Frontera G, Ruiz X, et al. Concomitant carcinoma in situ and tumour size are prognostic factors for bladder recurrence after nephroureterectomy for upper tract transitional cell carcinoma. BJU Int 2010;106:1319-23.

17 Wolbers M, Koller MT, Witteman JCM, et al. Prognostic models with competing risks: methods and application to coronary risk prediction. Epidemiology 2009;20:555-61.
18 Noordzij M, Leffondré K, van Stralen KJ, et al. When do we need competing risks methods for survival analysis in nephrology? Nephrol Dial Transplant 2013;28:2670-7.

19 He C, Zhang Y, Cai Z, et al. Overall survival and cancer-specific survival in patients with surgically resected pancreatic head adenocarcinoma: a competing risk nomogram analysis. J Cancer 2018;9:3156-67.

20 Yang J, Pan Z, He Y, et al. Competing-risks model for predicting the prognosis of penile cancer based on the seer database. Cancer Med 2019;8:7881-9.

21 Soria F, Shariat SF, Lerner SP, et al. Epidemiology, diagnosis, preoperative evaluation and prognostic assessment of upper-tract urothelial carcinoma (UTUC). World J Urol 2017;35:379-87.

22 Ludbrook J, Royse AG. Analysing clinical studies: principles, practice and pitfalls of Kaplan-Meier plots. ANZ J Surg 2008;78:204-10.

23 Putter H, Fiocco M, Geskus RB. Tutorial in biostatistics: competing risks and multi-state models. Stat Med 2007;26:2389-430.

24 Fine JP, Gray RJ. A proportional hazards model for the Subdistribution of a competing risk. J Am Stat Assoc 1999;94:496-509.

25 Kutikov A, Egleston BL, Canter D, et al. Competing risks of death in patients with localized renal cell carcinoma: a comorbidity based model. J Urol 2012;188:2077-83.

26 Bradshaw PT, Stevens J, Khankari N, et al. Cardiovascular disease mortality among breast cancer survivors. Epidemiology 2016;27:6-13.

27 Margulis V, Shariat SF, Matin SF, et al. Outcomes of radical nephroureterectomy: a series from the upper tract urothelial carcinoma collaboration. Cancer 2009;115:1224-33.

28 Yap SA, Schupp CW, Chamie K, et al. Effect of age on transitional cell carcinoma of the upper urinary tract: presentation, treatment, and outcomes. Urology 2011;78:87-92.

29 Leow JJ, Orsola A, Chang SL, et al. A contemporary review of management and prognostic factors of upper tract urothelial carcinoma. Cancer Treat Rev 2015;41:310-9.

30 Lughezzani G, Sun M, Perrotte P, et al. Gender-Related differences in patients with stage I to III upper tract urothelial carcinoma: results from the surveillance, epidemiology, and end results database. Urology 2010;75:321-7.

31 Espiritu PN, Sverrisson EF, Sexton WJ, et al. Effect of tumor size on recurrence-free survival of upper tract urothelial carcinoma following surgical resection. Urol Oncol 2014;32:619-24.

32 Freifeld Y, Krabbe L-M, Clinton TN, et al. Therapeutic strategies for upper tract urothelial carcinoma. Expert Rev Anticancer Ther 2018;18:765-74.

33 Seisen T, Krasnow RE, Bellmunt J, et al. Effectiveness of adjuvant chemotherapy after radical nephroureterectomy for locally advanced and/or positive regional lymph node upper tract urothelial carcinoma. $J$ Clin Oncol 2017;35:852-60.

34 Leow JJ, Martin-Doyle W, Fay AP, et al. A systematic review and meta-analysis of adjuvant and neoadjuvant chemotherapy for upper tract urothelial carcinoma. Eur Urol 2014;66:529-41.

35 Rouprêt M, Babjuk M, Compérat E, et al. European guidelines on upper tract urothelial carcinomas: 2013 update. Eur Urol 2013;63:1059-71.

36 Bolenz C, Fernández MI, Trojan L, et al. Lymphovascular invasion and pathologic tumor stage are significant outcome predictors for patients with upper tract urothelial carcinoma. Urology 2008;72:364-9.

37 Jin C, Deng X, Li Y, et al. Lymph node ratio is an independent prognostic factor for rectal cancer after neoadjuvant therapy: a metaanalysis. J Evid Based Med 2018;11:169-75.

38 Vinh-Hung V, Verkooijen HM, Fioretta G, et al. Lymph node ratio as an alternative to $\mathrm{pN}$ staging in node-positive breast cancer. $J$ Clin Oncol 2009;27:1062-8. 